\title{
Variación estacional y vertical de la biomasa del macrozooplancton en la bahía de Mochima, Estado Sucre - Venezuela, durante 1997 - 1998
}

\author{
Seasonal and vertical variation of macrozooplankton biomass in the Mochima Bay, \\ Sucre State, Venezuela, during 1997 - 1998

\section{Brightdoom Márquez ${ }^{1}$, Marín Baumar ${ }^{1}$ José Rafael Díaz-Ramos ${ }^{1}$, Luis Troccoli ${ }^{2}$ y Sonia Subero-Pino ${ }^{3}$}

\author{
${ }^{1}$ Departamento de Biología Marina, Laboratorio de Zooplancton. Instituto Oceanográfico de Venezuela, Universidad de Oriente. \\ Apdo. Postal 245. Cumaná. Sucre 6101. Venezuela \\ ${ }^{2}$ Escuela de Ciencias Aplicadas del Mar (ECAM), Universidad de Oriente, Núcleo de Nueva Esparta \\ ${ }^{3}$ Escuela de Ciencias, Dpto. Biología, Universidad de Oriente, Núcleo de Sucre, Apdo. Postal 19. Cumaná, Sucre 6101. Venezuela \\ bmarquez@sucre.udo.edu.ve
}

\begin{abstract}
The role of macrozooplankton in Mochima Bay was studied examining its biomass and its relationships with some environmental parameters during 1997 and 1998. Water transparency was measured with a Secchi disk; water samples were collected with a Niskin bottle $(5 \mathrm{~L})$ to determine: temperature, dissolved oxygen and phytoplankton biomass in two stations. Macrozooplankton horizontal samples were collected with a net (mesh size $555 \mu \mathrm{m}$ and $0.25 \mathrm{~m}$ in diameter) at $0-5$ and $10-15 \mathrm{~m}$ in two stations, one external (Est. A) and other internal (Est. B). Macrozooplankton biomass was estimated in terms of displaced volume and dry weight. The periods of maximum macrozooplankton biomass occurred between March-May and October-December for both years; the minima occurred in July-September. The highest biomass values were measured at the inner station and in the deeper layer $(10-15 \mathrm{~m})$. Maximum values for phytoplankton and macrozooplankton biomass coincided with periods of higher upwelling and wind intensity; these values were higher in 1997 than in 1998. The results follow preestablished patterns but also show high annual variability in phytoplankton and zooplankton biomass.
\end{abstract}

Key words: Zooplankton, biomass, production, Mochima Bay
Resumen.- El papel del macrozooplancton en la bahía de Mochima fue determinado cuantitativamente mediante su biomasa y su relación con algunos parámetros ambientales durante 1997 y 1998. Se midió la transparencia del agua con un disco de Secchi; las muestras de agua fueron recolectadas con una botella Niskin ( 5 L) para determinar: temperatura, oxígeno disuelto y biomasa fitoplanctónica en dos estaciones. Las muestras de macrozooplancton fueron recolectadas por medio de calados oblicuos, con una malla estándar $(555 \mu \mathrm{m}$ y $0,25 \mathrm{~m}$ de diámetro de boca) entre 0-5 y 10-15 m, en dos estaciones, una externa (Est. A) y otra interna (Est. B). La biomasa se estimó en términos del volumen desplazado y del peso seco. Los períodos de máxima biomasa macrozooplanctónica se registraron entre marzo-mayo y octubre-diciembre para ambos años; los mínimos ocurrieron en julio-septiembre. La mayor abundancia se detectó en la estación interna y en la capa profunda (10-15 m). Los máximos valores de la biomasa de fito y macrozooplancton coincidieron con períodos de mayor intensidad de la surgencia costera e intensidad del viento. Estos valores fueron más altos en 1997 que en 1998. El patrón estacional de la biomasa planctónica es similar al señalado para la zona. Sin embargo, se demuestra la existencia de una alta variabilidad anual en el crecimiento del fito y del macrozooplancton.

Palabras clave: Zooplancton, biomasa, producción, bahía de Mochima

\section{Introducción}

El zooplancton retiene y transfiere la materia y energía que el fitoplancton ha fijado fotosintéticamente, y por tanto es un indicador de la producción secundaria (Fernández de Puelles 1990, Clark et al. 2001). En los estudios del zooplancton, la determinación de la biomasa es muy importante y puede complementar la información de la composición, abundancia y estructura comunitaria (Gasca \& Suárez 1996). La producción de un determinado nivel trófico marino se define por la cantidad de materia orgánica sintetizada por unidad de tiempo (Postel et al. 2000). Aunque la 
biomasa de una comunidad mide condiciones instantáneas, puede usarse como medida de producción de biomasa, ya que el análisis temporal y frecuente brinda una estimación de la abundancia y conocimiento de su comportamiento, evolución y dinámica. Asimismo, al ser un parámetro estandarizado, permite comparaciones geográficas (Fernández de Puelles \& García-Braun 1989).

Las fluctuaciones temporales en la biomasa del zooplancton no responden a fenómenos aislados, sino que van ligadas a estructuras de naturaleza trófica (fitoplancton) e hidrodinámicas (estructura térmica, salina, turbulencias y advección). De la relación de todos estos parámetros se puede comprender mejor la dinámica del ecosistema y aquellos factores que tienen mayor influencia sobre el equilibrio y estabilidad del área (Guzmán \& Obando 1988, Fernández de Puelles \& García-Braun 1989, Fernández de Puelles 1990).

En Venezuela, los estudios sobre la biomasa zooplanctónica son descriptivos y la mayoría ha sido realizado en la región nororiental del país dentro de la cual se encuentra la bahía de Mochima $\left(10^{\circ} 24^{\prime}-10^{\circ}\right.$ $20^{\prime} \mathrm{N}$ y $64^{\circ} 19^{\prime} 30^{\prime \prime}-64^{\circ} 22^{\prime} 30^{\prime \prime}$ W). La bahía ubicada dentro del parque nacional del mismo nombre, es uno de los ecosistemas costeros favorecidos por procesos de surgencia costera. Las condiciones físicas y químicas del agua de esta bahía han sido estudiadas por varios autores (Okuda et al. 1968, Kato 1972, García 1978) quienes señalan que los parámetros ambientales muestran cierta estacionalidad relacionada con los períodos de sequía y lluvia. La abundancia y biomasa del zooplancton de la bahía aumentan durante la época de surgencia, originando así una zona fértil con un gran potencial pesquero. En ella se han realizado los trabajos de zooplancton sobre: la distribución de misidáceos en manglares y arrecifes coralinos (Zoppi et al. 1989, Zoppi \& Alonso 1997) y el zooplancton de la zona interna (Zoppi 1999); mientras que en fitoplancton se pueden citar los de González \& Zoppi (1997) y Expósito \& Zoppi (1999) quienes estudiaron la comunidad fitoplanctónica cercana al punto de descarga de la laguna de estabilización de esa localidad. A fin de conocer el papel del macrozooplancton en la bahía de Mochima, en este trabajo se estima cuantitativamente su producción en forma de volumen desplazado y peso seco, a lo largo de dos años, y se relacionan estos resultados con alguna de las variables hidrológicas (temperatura, salinidad, oxígeno, clorofila a), bajo la premisa de que estos pueden causar variaciones importantes en la biomasa macrozooplanctónica.

\section{Material y métodos}

Se realizaron los muestreos mensualmente desde el 29 de enero, 1997 al 18 de diciembre, 1998, entre las 8:00 y 10:00 a.m. Se escogieron dos estaciones, una externa (A) y otra interna (B) ubicadas en la entrada y en la parte interna de la bahía, respectivamente (Fig. 1).

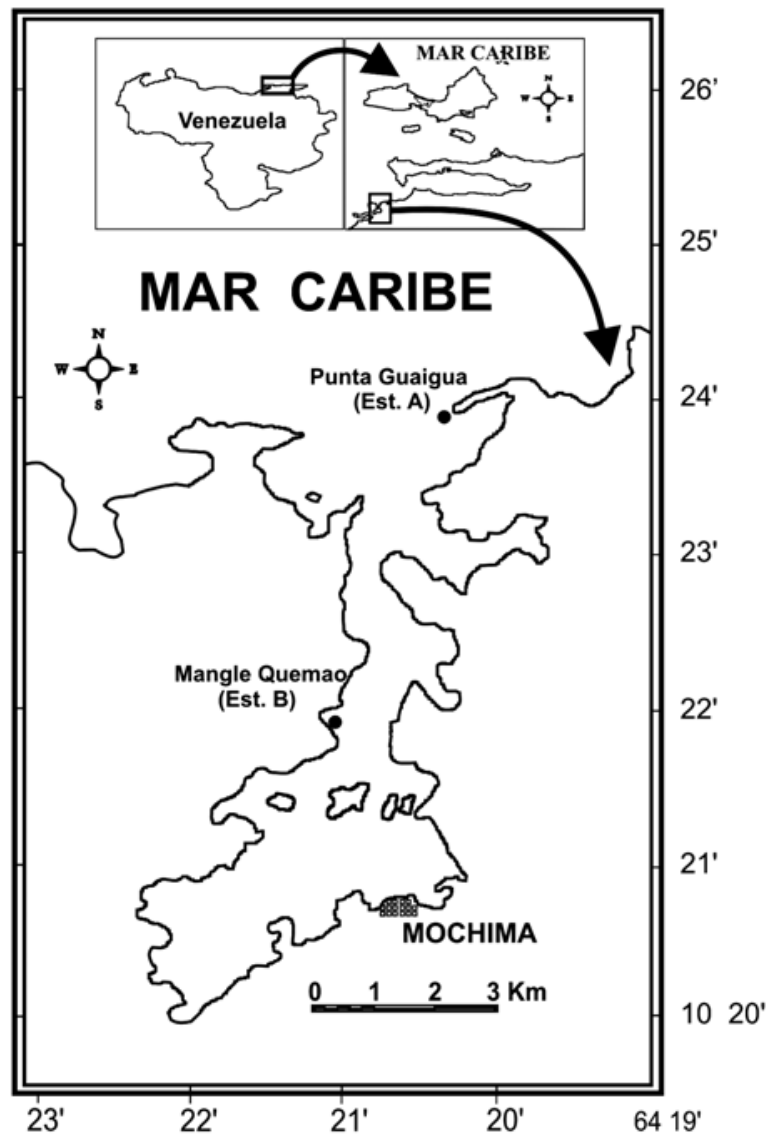

Figura 1

Mapa de la bahía de Mochima mostrando la ubicación de las dos estaciones de muestreo

Map of the Mochima Bay showing the location of the two sampling stations 
La transparencia del agua se midió con un disco de Secchi. Se utilizó una botella de Niskin $(5 \mathrm{~L})$ para recolectar muestras de agua a 0,5 y $10 \mathrm{~m}$ de profundidad. Se determinó la concentración de oxígeno disuelto por el método de Winkler (Strickland \& Parsons 1972), la temperatura con un termómetro ambiental $\left( \pm 0,1^{\circ} \mathrm{C}\right)$, la concentración de clorofila $a$ por el método de Lorenzen modificado por Strickland \& Parsons (1972) y la salinidad por un refractómetro. Se utilizó una red de malla tipo estándar de $(555 \mu \mathrm{m})$ y $0,25 \mathrm{~m}$ de diámetro de boca para realizar calados oblicuos, a dos profundidades (0-5 y 10-15 m), y a una velocidad de 2 nudos durante 10 minutos. El cálculo del volumen de muestreo se estimó a través de la ecuación $\mathrm{V}=\pi \mathrm{r}^{2} \mathrm{~d}$ (1), donde, $\mathrm{r}$ es el radio de la boca de la red y d es la distancia recorrida; la distancia recorrida durante el calado se calculó mediante $\mathrm{d}=\mathrm{v} \mathrm{t}$ (2), donde $\mathrm{v}$ es la velocidad de la embarcación y t el tiempo que se utilizó para el calado. Se obtuvo un volumen aproximado de 101,54 $\mathrm{m}^{3}$.

Las muestras de zooplancton se fijaron con formaldehído (4\%) neutralizado con borato de sodio. Cada muestra se homogeneizó y se dividió en dos porciones iguales por medio de un separador de Folsom (McEven et al. 1954), una porción se utilizó para estudiar la estructura de la comunidad del zooplancton y la otra para los análisis de biomasa volumétrica (desplazada) y gravimétrica (peso seco), siguiendo la metodología detallada en Beers (1981) y Postel et al. (2000). Estos valores se multiplicaron por dos para estimar el valor total de la muestra.
La velocidad $\left(\mathrm{m} \mathrm{s}^{-1}\right)$ y dirección de los vientos (grados) fueron tomados de la Estación Metereológica del Aeropuerto Antonio José de Sucre, a unos $25 \mathrm{~km}$ al este de la bahía. El índice de surgencia (IS) fue calculado por la fórmula IS $=\mathrm{t}_{\mathrm{sx}} / \mathrm{rw}$ f 100 (3); donde rw es la densidad del agua (cte) $=1025 \mathrm{~kg} \mathrm{~m}^{-3} ; \mathrm{f}=$ parámetro de Coriolis (cte) $=2 \mathrm{w}$ sen fin $=2,64 \cdot 10^{-7}$; tsx $=$ fricción sup. del viento sería tsx $=\mathrm{k} \mathrm{ra}^{2}$ (4), donde $\mathrm{k}=$ coeficiente empírico de dragado (cte) = $1,11($ si $\mathrm{W}<5)$ y $1,45($ si $\mathrm{W}>5)$; ra = densidad media del aire $($ cte $)=122 \times 10^{-8} \mathrm{~kg} \mathrm{~cm}^{-3} ; \mathrm{w}=$ velocidad del viento (Bowden 1983).

Para determinar posibles diferencias entre el efecto de la profundidad y el tiempo sobre la biomasa zooplanctónica en cada estación, se empleó un análisis de varianza de dos factores sin réplica (Sokal \& Rohlf 1981). Los datos se transformaron a $\log (\mathrm{x}+1)$ para cumplir con las suposiciones de normalidad $y$ homogeneidad de varianzas (Sokal \& Rohlf 1981). Para establecer las relaciones entre las variables hidrográficas y biomasa fitoplanctónica (clorofila) y del zooplancton en cada año de estudio, se realizó un análisis de componentes principales (ACP) a partir de una matriz de correlación (Johnson \& Wichern 1992).

\section{Resultados}

\section{Factores abióticos}

Los estadísticos básicos de los parámetros estudiados se presentan en la Tabla 1. El índice de surgencia (IS) presentó valores superiores a $1000 \mathrm{~m}^{3} \mathrm{~s}^{-1} 1000 \mathrm{~m}$ durante casi todo el primer semestre de 1997 (Fig. 2A).

Tabla 1

Estadísticos básicos de los parámetros bióticos y abióticos medidos en la bahía de Mochima, durante 1997 y 1998

Basic statistics of the biotic and abiotic measured parameters of the Mochima Bay, during 1997 and 1998

\begin{tabular}{|c|c|c|c|c|c|c|}
\hline Año & Parámetro & $\mathrm{n}$ & Promedio & D. E. & Mínimo & Máximo \\
\hline \multirow[t]{6}{*}{1997} & Transparencia (m) & 24 & 11 & 3,70 & 4 & 19 \\
\hline & Temperatura $\left({ }^{\circ} \mathrm{C}\right)$ & 96 & 24,8 & 2,1 & 20,3 & 29,3 \\
\hline & Oxígeno disuelto $\left(\mathrm{mL} \mathrm{L}^{-1}\right)$ & 24 & 4,38 & 0,49 & 3,64 & 5,28 \\
\hline & Biomasa fitoplanctónica (mg chl $a \mathrm{~m}^{-3}$ ) & 91 & 1,260 & 1,576 & 0,118 & 8,872 \\
\hline & Volumen desplazado $\left(\mathrm{mL} \mathrm{m}^{-3}\right)$ & 48 & 0,208 & 0,168 & 0,040 & 0,797 \\
\hline & Peso seco $\left(\mathrm{mg} \mathrm{m}^{-3}\right)$ & 48 & 0,039 & 0,017 & 0,018 & 0,088 \\
\hline \multirow[t]{6}{*}{1998} & Transparencia & 24 & 13 & 2,0 & 3,2 & 8,5 \\
\hline & Temperatura $\left({ }^{\circ} \mathrm{C}\right)$ & 96 & 26,5 & 2,5 & 20,8 & 31 \\
\hline & Oxígeno disuelto $\left(\mathrm{mL} \mathrm{L}^{-1}\right)$ & 24 & 4,07 & 0,56 & 2,95 & 5,48 \\
\hline & Biomasa fitoplanctónica (mg chl $a \mathrm{~m}^{-3}$ ) & 83 & 0,802 & 0,871 & 0 & 4,272 \\
\hline & Volumen desplazado $\left(\mathrm{mL} \mathrm{m}^{-3}\right)$ & 48 & 0,061 & 0,053 & 0,019 & 0,299 \\
\hline & Peso seco $\left(\mathrm{mg} \mathrm{m}^{-3}\right)$ & 48 & 0,017 & 0,007 & 0,009 & 0,043 \\
\hline
\end{tabular}




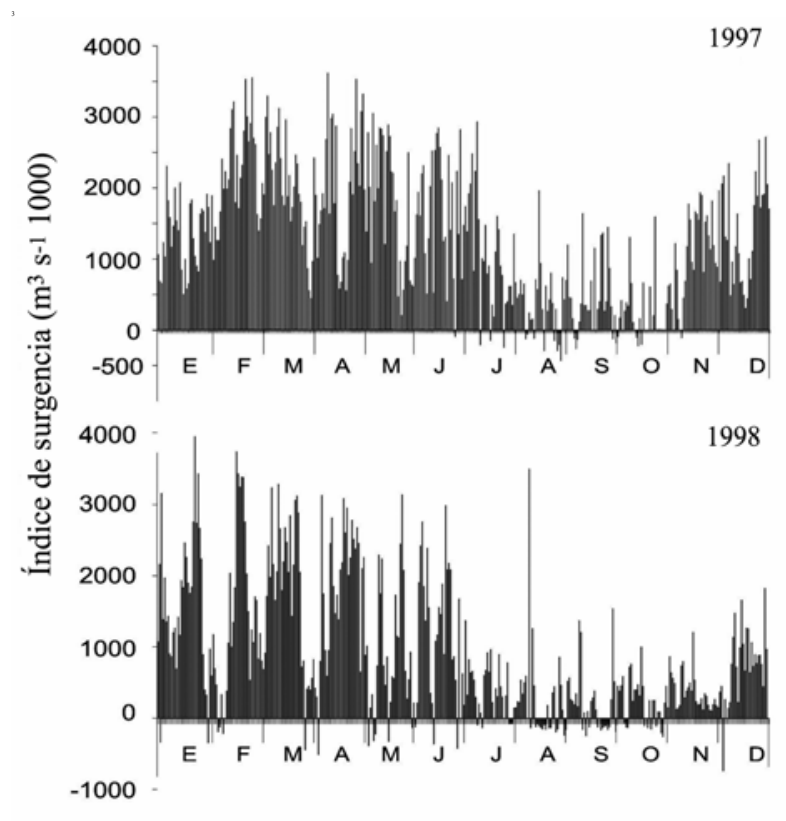

Figura 2

Índice de surgencia $\left(\mathrm{m}^{3} \mathrm{~s}^{-1} 1000 \mathrm{~m}\right)$ en la bahía de Mochima, Venezuela, durante 1997 y 1998

Upwelling index $\left(\mathrm{m}^{3} \mathrm{~s}^{-1} 1000 \mathrm{~m}\right)$ in Mochima Bay, Venezuela, during 1997 and 1998

Entre julio y noviembre, el índice de surgencia exhibió valores inferiores a $500 \mathrm{~m}^{3} \mathrm{~s}^{-1} 1000 \mathrm{~m}$ e incluso se registraron valores negativos en agosto, septiembre y octubre. Al final del año, los valores tendieron a ser superiores $\left(>700 \mathrm{~m}^{3} \mathrm{~s}^{-1} 1000 \mathrm{~m}\right)$ a los registrados en el período anterior. En 1998, el IS se caracterizó por una disminución en los períodos de alta intensidad y fuertes oscilaciones (Fig. 2B); aparecieron con mayor frecuencia valores negativos a lo largo de este año con respecto a 1997, extendiéndose el período de baja intensidad desde junio a diciembre.

La transparencia del agua varió de manera similar en las dos estaciones (Fig. 3); sin embargo, los valores tendieron a ser mayores en la estación externa (Est. A). Los menores valores se midieron en febrero y marzo en 1997 y de enero a abril en 1998, mientras que los mayores valores se registraron en la segunda mitad del año excepto el pico medido en enero y junio 1997 en las dos estaciones. En ambas estaciones, la transparencia del agua fue mayor en 1998 que en 1997 (Tabla 1).

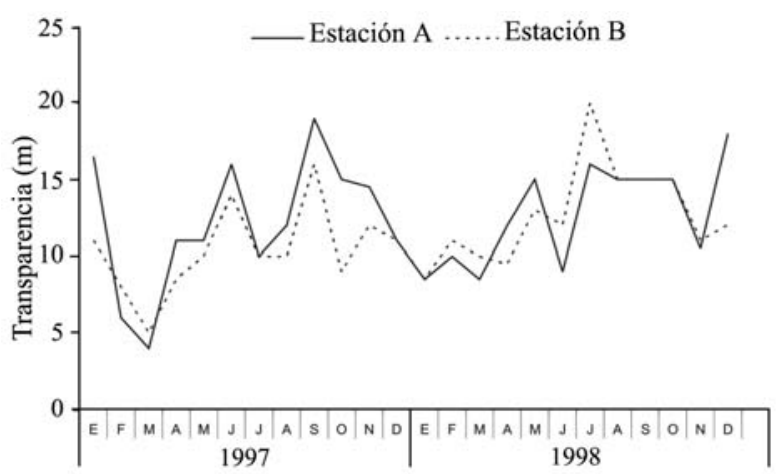

Figura 3

Variación mensual de la transparencia del agua en la bahía de Mochima, Venezuela, durante 1997 y 1998

Monthly variation in water transparency in Mochima Bay, Venezuela, during 1997 and 1998

Al igual que la transparencia, la variación temporal de la temperatura del agua fue similar en las dos estaciones (Figs. 4A y 5A). Las diferencias térmicas entre la superficie y $10 \mathrm{~m}$ de profundidad fueron más marcadas en la parte interna (B) que en la boca (A). También se notó que la columna de agua registró menor temperatura en 1997 que en 1998 (Tabla 1). En ambos años y para ambas estaciones, la temperatura del agua disminuyó entre enero y marzo. En el período abril-junio se incrementó la temperatura en la columna de agua. Este proceso de calentamiento se interrumpió en julio 1997 pero no así en julio 1998. Los máximos valores de temperatura $\left(>27^{\circ} \mathrm{C}\right)$ se midieron entre agosto y octubre en ambas estaciones. A finales de año, la temperatura de la columna de agua disminuyó en ambas estaciones.

La concentración de oxígeno disuelto varió notablemente con respecto a la profundidad en las dos estaciones durante el estudio (Figs. 4B y 5B). En la estación $\mathrm{A}$, los valores superficiales tendieron a ser más altos (que en el resto de la columna de agua durante los meses donde disminuyó la temperatura) mientras que en los meses de aguas cálidas, los valores fueron similares o menores a los medidos a $5 \mathrm{~m}$ o incluso inferiores a los medidos a $10 \mathrm{~m}$. Las menores concentraciones de oxígeno disuelto se midieron a 10 $\mathrm{m}$ durante los meses con aguas cuya temperatura fue menor a $24^{\circ} \mathrm{C}$. 


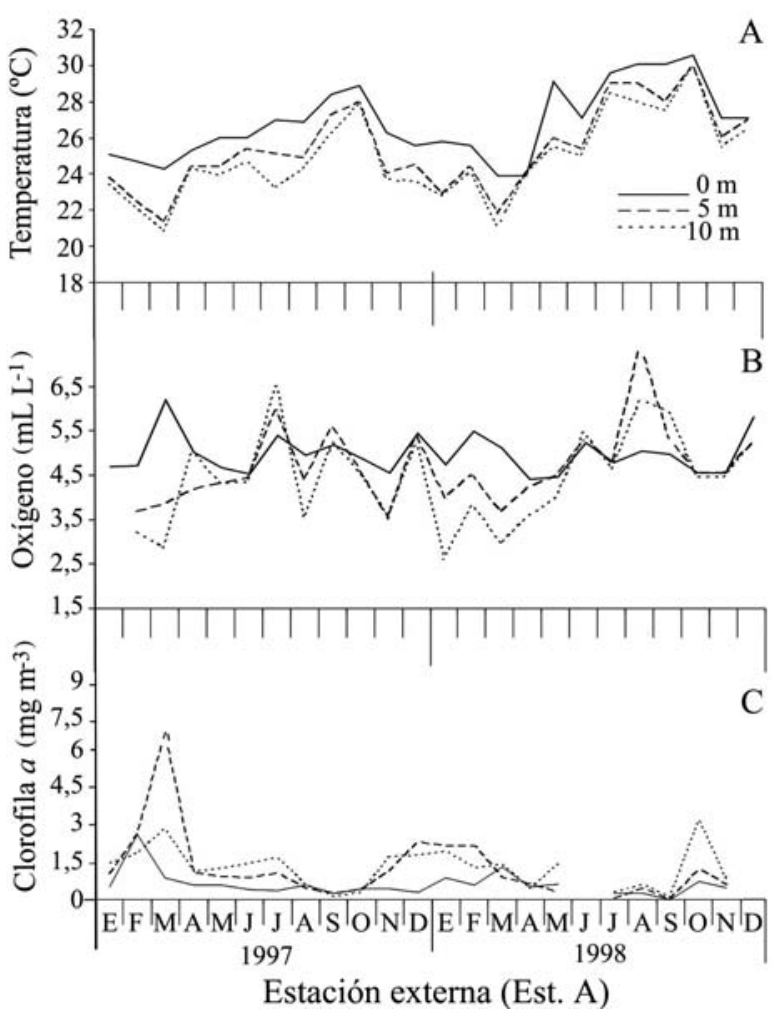

Figura 4

Variación mensual de la temperatura (A), oxígeno disuelto (B) y clorofila a (C) en la estación externa (Est. A) en la bahía de Mochima, Venezuela, durante 1997 y 1998

Monthly variation in temperature (A), dissolved oxygen (B), and chlorophyll $a$ concentration $(\mathrm{C})$ in the external station

(Est. A), in Mochima Bay, Venezuela, during 1997 and 1998

Por otra parte, en la estación B, los valores de oxígeno disuelto en la superficie y $5 \mathrm{~m}$ son superiores a $4,4 \mathrm{mLO}_{2} \mathrm{~L}^{-1}$ y varían de manera similar durante el estudio (Fig. 5B). Los mayores cambios temporales se produjeron a $10 \mathrm{~m}$. En los meses con temperaturas más bajas $\left(<24^{\circ} \mathrm{C}\right)$, los valores a $10 \mathrm{~m}$ fueron menores a 4,0 $\mathrm{mLO}_{2} \mathrm{~L}^{-1}$. Es importante señalar, tal como se observa en zonas de influencia de la surgencia, que en los meses de aguas cálidas $\left(>24^{\circ} \mathrm{C}\right)$, el valor de este parámetro tendió a ser superior a $4,5 \mathrm{mLO}_{2} \mathrm{~L}^{-1}$ y $\mathrm{su}$ distribución vertical tendió a ser uniforme. También, hay que resaltar el hecho de que las diferencias batimétricas con respecto al oxígeno disuelto son mayores en la estación A que en la B.

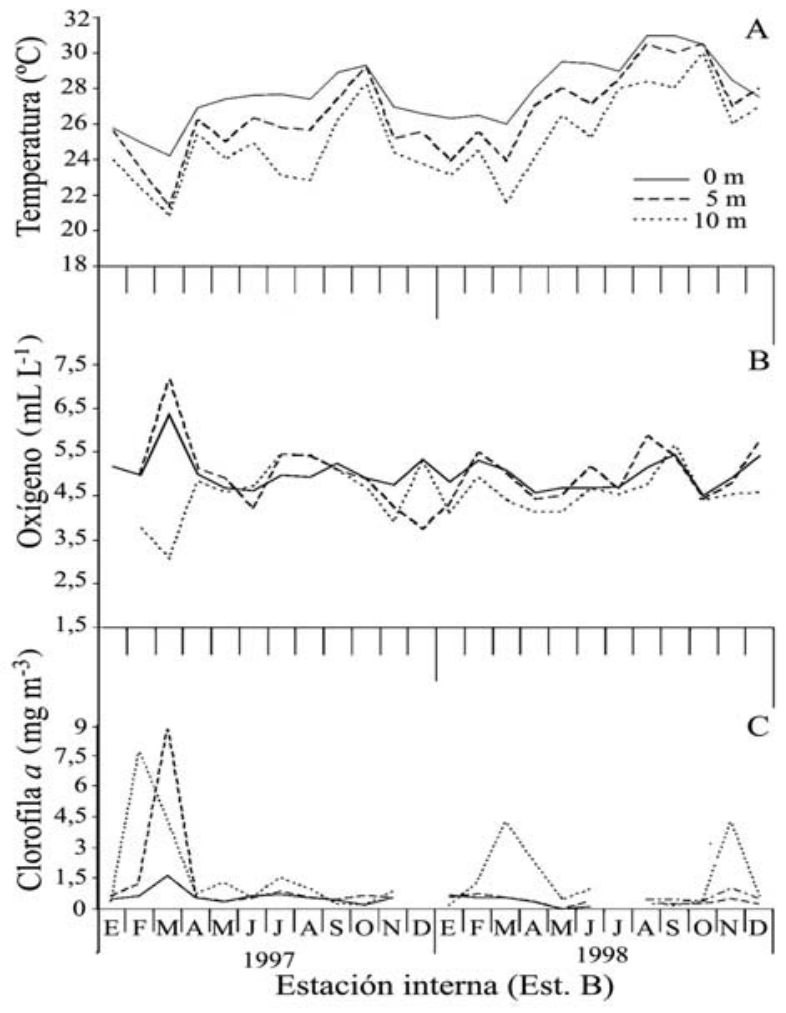

Figura 5

Variación mensual de la temperatura (A), oxígeno disuelto (B) y clorofila $a$ (C) en la estación interna (Est. B) en la bahía de Mochima, Venezuela, durante 1997 y 1998

Monthly variation in temperature (A), dissolved oxygen (B), and chlorophyll $a$ concentration $(\mathrm{C})$ in the internal station

(Est. B) in Mochima Bay, Venezuela, during 1997 and 1998

Los valores de salinidad mostraron alta estabilidad, en las dos estaciones, registrándose valores entre 36,00 y 37,00 ups durante el estudio.

\section{Factores bióticos}

En la estación A, la concentración de chl $a$ en la superficie fue menor $\left(<1,5 \mathrm{mg}\right.$ chl $\left.a \mathrm{~m}^{-3}\right)$ que en las profundidades restantes excepto en febrero, agostooctubre 1997 y septiembre-octubre 1998 (Fig. 4C). Los valores máximos de biomasa fitoplanctónica $(>1,5 \mathrm{mg}$ chl $a \mathrm{~m}^{-3}$ ) se midieron en: enero, febrero, julio, $\mathrm{y}$ noviembre 1997, febrero 1998 y en septiembre y octubre 1998. En esos meses, los valores más elevados se midieron a 5 o $10 \mathrm{~m}$. Gráficamente se pudo observar 
que durante los meses con bajas concentraciones de chl a (abril, mayo y octubre 1997; abril, julioseptiembre 1998), las diferencias entre profundidades fueron reducidas.

Al igual que en la estación $A$, la biomasa del fitoplancton superficial en la estación B fue menor que la medida en las profundidades restantes (Fig. 5C). En 1997 se midieron concentraciones altas de chl $a,(>1,5$ $\mathrm{mg}$ chl $a \mathrm{~m}^{-3}$ ) de febrero a marzo, en mayo y de julio a agosto. Por otra parte, en 1998, los valores máximos se midieron a $10 \mathrm{~m}$, de febrero a abril y en noviembre; sin embargo, estos valores fueron menores a los registrados en 1997 (Tabla 1). De nuevo, en los meses con bajas concentraciones de chl $a$ (abril, septiembre - noviembre 1997, mayo, agosto-octubre, diciembre 1998), las diferencias entre profundidades fueron mínimas, a diferencia de los meses de febrero y marzo de 1997 cuando hubo una marcada diferencia entre las profundidades $\left(1,5 \mathrm{mg} \mathrm{chl} a \mathrm{~m}^{-3}\right)$ y en las aguas más profundas entre (7-9 $\left.\mathrm{mg} \mathrm{chl} a \mathrm{~m}^{-3}\right)$.

En la estación externa (Est. A), las medidas de biomasa zooplanctónica expresadas en volumen sedimentado $\left(\mathrm{mL} \mathrm{m}^{-3}\right)$ presentaron una variación temporal similar; en 1997 la variación fue bimodal (Fig. 6). Fue notorio el hecho de que durante los meses con altos valores de biomasa, los valores medidos en el nivel profundo fueron mayores $\left(0,38 \mathrm{~mL} \mathrm{~m}^{-3}\right)$ que los medidos en la superficie $\left(0,15 \mathrm{~mL} \mathrm{~m}^{-3}\right)$, mientras que en los meses con baja biomasa las diferencias entre profundidades fueron mínimas. Los valores máximos del volumen sedimentado a nivel superficial fueron en abril de 1997 y 1998, así como en julio de 1997 y octubre de 1998; sin embargo, a nivel profundo, las magnitudes fueron superiores, alcanzando valores por encima de $0,25 \mathrm{~mL} \mathrm{~m}^{-3}$ en abril y junio de $1997 \mathrm{y}$ noviembre de 1997 y 1998 (Fig. 6A). En cuanto al peso seco, se produjeron diferencias con respecto a la profundidad en octubre y noviembre de 1997 y de 1998 (Fig. 6B). Las diferencias interanuales fueron notorias. En 1997 se midieron los valores más altos de biomasa $\mathrm{y}$, en general, los valores fueron más altos que en 1998 (Tabla 1). En 1997 la abundancia fue alta entre marzo y mayo y/o junio ( $>0,20 \mathrm{~mL} \mathrm{~m}^{-3}$, volumen desplazado; $>0,030 \mathrm{mg} \mathrm{m}^{-3}$, de peso seco) y en noviembre, mientras que en 1998, la biomasa zooplanctónica fue baja todo el año. Se encontraron diferencias altamente significativas de la biomasa zooplanctónica entre los dos años de estudio y entre las profundidades ( $\mathrm{Fs}=$ $16,87 ; P \leq 0,001)$.

Al igual que la tendencia de la estación externa, en la estación interna (Est. B), los valores de biomasa del zooplancton del nivel profundo fueron más elevados que los superficiales (Fig. 7). Sin embargo, los resultados de las mediciones de biomasa en la estación interna difirieron de la externa en varios aspectos (Figs. 6 y 7$)$.

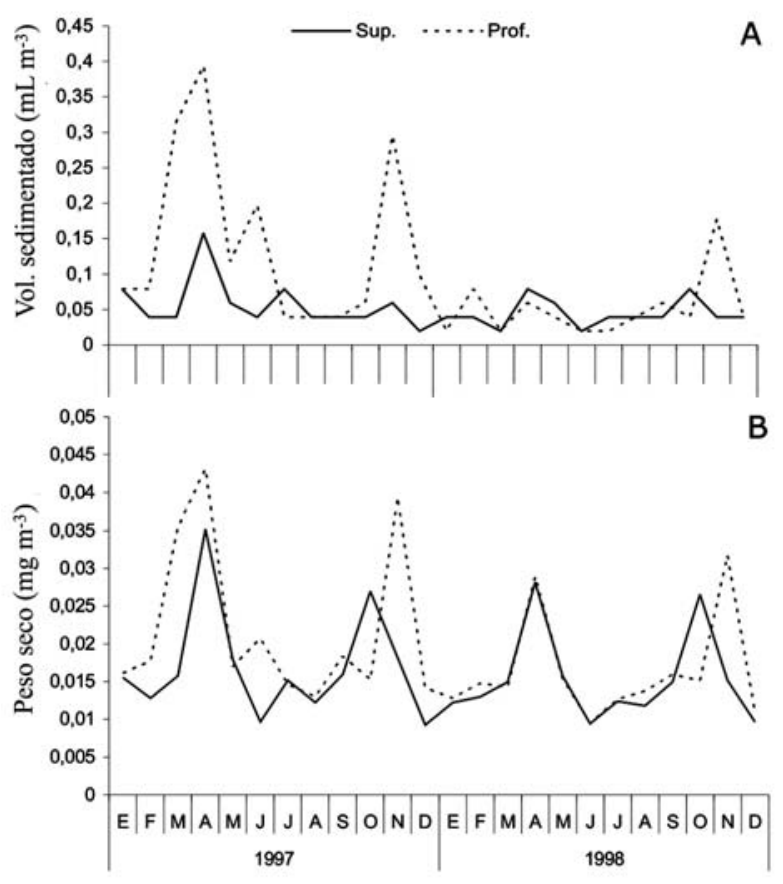

Estación externa (Est. A)

Figura 6

Variación anual de la biomasa del macrozooplancton de la estación externa (Est. A) en la bahía de Mochima, Venezuela, durante 1997 y 1998. (A) volumen desplazado $\left(\mathrm{mL} \mathrm{m}^{-3}\right)$ y (B) peso seco $\left(\mathrm{mg} \mathrm{m}^{-3}\right)$

Annual variation in the macrozooplankton biomass at the external station (Est. A) in Mochima Bay, Venezuela, during 1997 and 1998. (A) displaced volume $\left(\mathrm{mL} \mathrm{m}^{-3}\right)$ and (B) dry weight $\left(\mathrm{mg} \mathrm{m}^{-3}\right)$ 


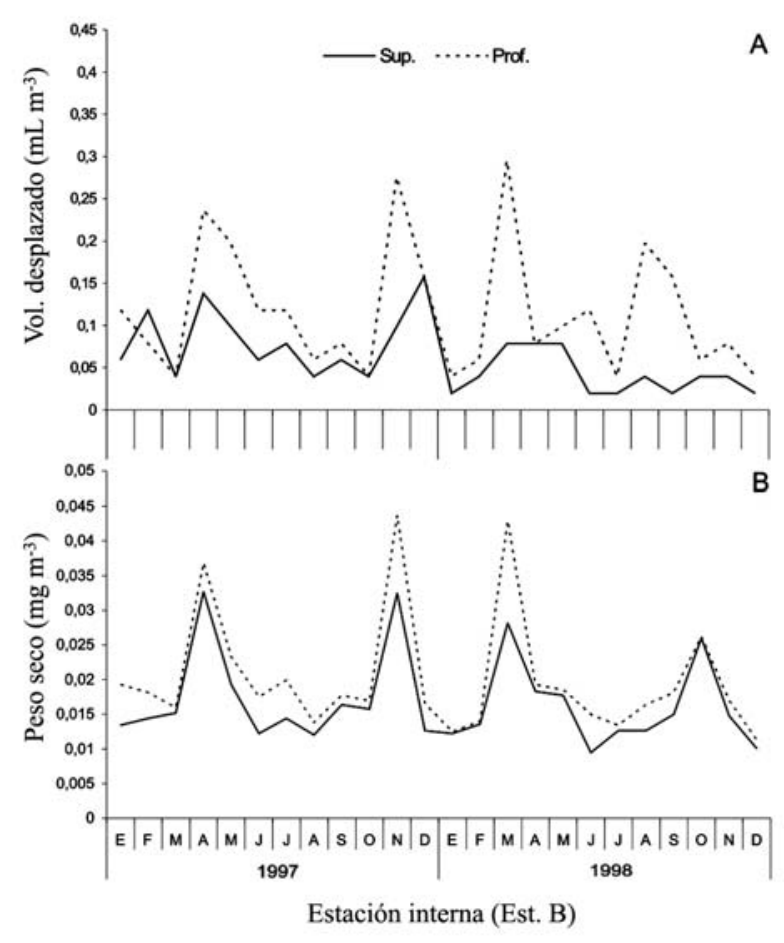

Figura 7

Variación anual de la biomasa del macrozooplancton de la estación interna (Est. B) en la bahía de Mochima, Venezuela, durante 1997 y 1998. (A) volumen desplazado $\left(\mathrm{mL} \mathrm{m}^{-3}\right)$ y (B) peso seco $\left(\mathrm{mg} \mathrm{m}^{-3}\right)$

Annual variation in the macrozooplanktonic biomass at the internal station (Est. B) in Mochima Bay, Venezuela, during 1997 and 1998. (A) displaced volume $\left(\mathrm{mL} \mathrm{m}^{-3}\right)$ and (B) dry weight $\left(\mathrm{mg} \mathrm{m}^{-3}\right)$

Primero, en la estación interna, las medidas de biomasa mostraron tres o cuatro períodos de valores altos en 1997 y dos en 1998. Segundo, se produjeron diferencias notorias entre el volumen desplazado superficial y profundo; esto debido posiblemente a la presencia de organismos gelatinosos y del cladócero Penilia avirostris identificados en las muestras; estas diferencias en los valores con respecto a la profundidad no se produjeron en el peso seco, ni tampoco en las mediciones hechas en la estación externa. Tercero, las mediciones hechas en 1998, en la estación interna (Est. B) fueron más altas que las de la externa; de hecho se presentaron máximos en marzo y agosto-septiembre y octubre mientras que en la estación externa estos sólo se observaron en noviembre. Los valores de biomasa variaron de manera significativa con respecto a la
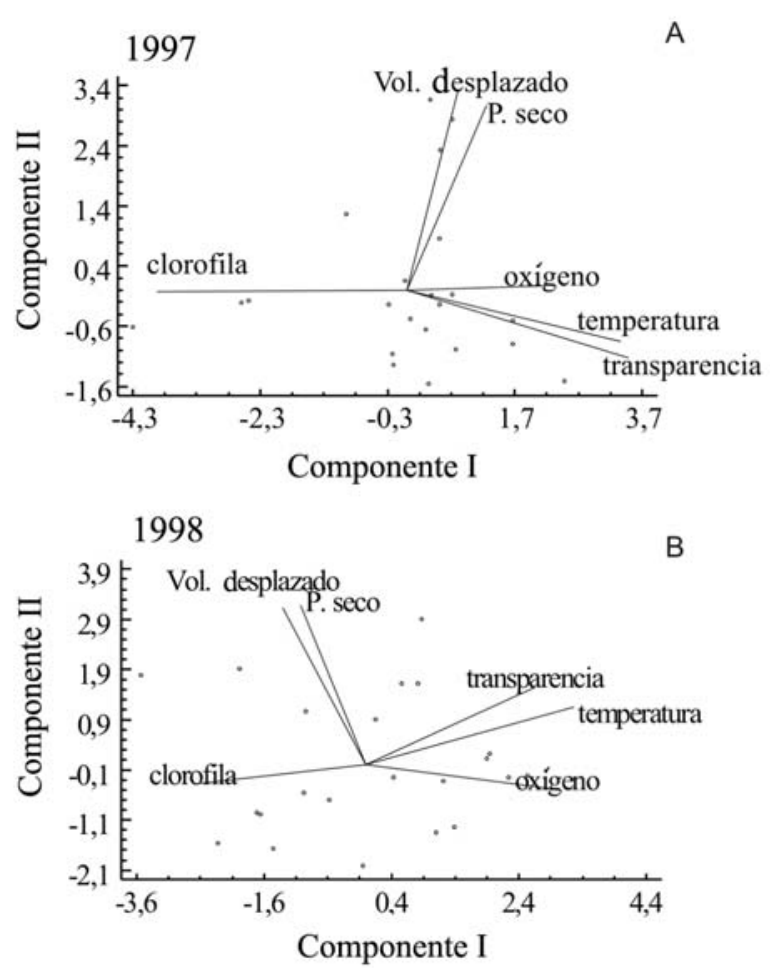

Figura 8

Proyección ortogonal de los dos primeros componentes de las variables hidrológicas e hidrobiológicas de la bahía de Mochima. (A) 1997 y (B) 1998

Orthogonal projections of the first two components of hydrological and hydrobiological variables in Mochima Bay. (A) 1997 and (B) 1998

profundidad, siendo mayores en el nivel profundo ( $\mathrm{Fs}=$ 9,05; $P \leq$ 0,01). En 1998, los valores obtenidos por los dos métodos, fueron más bajos que los de 1997 para ambas profundidades, apreciándose diferencias altamente significativas entre los mismos $(\mathrm{Fs}=16,87 ; P \leq 0,001)$.

El análisis de componentes principales (ACP) realizado a las variables hidrológicas e hidrobiológicas de la zona de estudio mostró una varianza acumulada de $82,07 \%$ en 1997 y 88,21\% en 1998 , en los tres primeros componentes (Tablas 2 y 3). Las Figs. $8 \mathrm{~A}$ y $8 \mathrm{~B}$ muestran las correlaciones significativas y negativas entre la temperatura del agua, transparencia, oxígeno disuelto y la biomasa del fitoplancton (componente 1) y altamente significativas y positivas entre el volumen desplazado y el peso seco (componente 2) para los dos años de estudio. En general, las tendencias de las 
Tabla 2

Porcentajes de varianza de los tres primeros componentes del análisis de componentes principales en la bahía de Mochima durante 1997 y 1998

Variance percentages of the first three components of the Principal Components Analysis in the Mochima Bay during 1997 and 1998

\begin{tabular}{ccc}
\hline Componente & $\mathbf{1 9 9 7}$ & $\mathbf{1 9 9 8}$ \\
\hline C-I & 37,40 & 45,39 \\
C-II & 28,96 & 30,25 \\
C-III & 15,70 & 12,56 \\
Total & $\mathbf{8 2 , 0 7}$ & $\mathbf{8 8 , 2 1}$ \\
\hline
\end{tabular}

Tabla 3

Correlaciones de los parámetros bióticos y abióticos con los tres primeros componentes en la bahía de Mochima durante el año 1997 (A) y el año 1998 (B)

Biotic and abiotic parameters correlations with the first three components in Mochima Bay during 1997 (A) and 1998 (B)

(A)

\begin{tabular}{lccc}
\hline \multicolumn{1}{c}{ Parámetros } & Componente I & Componente II & Componente III \\
\hline Temperatura & 0,54 & 0,23 & $-0,18$ \\
Oxígeno disuelto & 0,50 & $-0,01$ & 0,47 \\
Clorofila $a$ & $-0,44$ & $-0,08$ & 0,74 \\
Transparencia & 0,47 & 0,31 & 0,44 \\
Volumen desplazado & $-0,17$ & 0,65 & $-0,01$ \\
Peso seco & $-0,22$ & 0,64 & 0,02 \\
\hline
\end{tabular}

(B)

\begin{tabular}{lccc}
\hline \multicolumn{1}{c}{ Parámetros } & Componente I & Componente II & Componente III \\
\hline Temperatura & 0,50 & 0,18 & 0,45 \\
Oxigeno disuelto & 0,32 & $-0,01$ & $-0,86$ \\
Clorofila $a$ & $-0,58$ & 0,01 & $-0,02$ \\
Transparencia & 0,51 & 0,24 & 0,01 \\
Volumen desplazado & 0,12 & $-0,70$ & $-0,09$ \\
Peso seco & 0,19 & $-0,65$ & 0,24 \\
\hline
\end{tabular}

variables fueron las mismas para los dos años. En ambos casos la relación fitoplancton (chl a) y zooplancton no mostraron correlaciones significativas, tanto para componente 1 vs componente 2 como componente 2 vs componente 3 (Tabla 3 ).

\section{Discusión}

Las variaciones en la biomasa de macrozooplancton de ambas estaciones muestreadas, fueron similares con respecto a la profundidad y se asocian con los períodos de surgencia característicos de la zona nororiental de
Venezuela (Richards 1960, Okuda et al. 1968, Griffiths \& Simpson 1972, Moigis 1986, Lodeiros \& Himmelman 1994). Entre marzo-mayo y noviembrediciembre cuando se produjeron los períodos de mayor intensidad de la surgencia y del viento, se midieron los máximos valores de biomasa. Además, se encontraron niveles elevados de clorofila $a$ y baja concentración de oxígeno, coincidiendo con lo señalado por Mandelli \& Ferraz-Reyes (1982) y Ferraz-Reyes (1987). También se detectó un segundo período caracterizado por valores de biomasa intermedios que coincidió con un segundo período de surgencia de menor intensidad. 
Este fenómeno también ha sido observado por Rueda (2000). Por último, los menores valores de biomasa macrozoplanctónica se obtuvieron en el periodo agostooctubre, y se asocian a una disminución de la concentración de clorofila $a$, un aumento de la temperatura y aparición de las precipitaciones combinados con la disminución de la velocidad del viento y del índice de surgencia. Estas condiciones hidrográficas son características del período de estratificación o estancamiento en el oriente venezolano (Okuda et al. 1968, Okuda \& Benítez 1974). Urosa (1977) y Peñuela (2000) también midieron valores bajos de biomasa zooplanctónica en este período para el mesozooplancton. Es importante señalar que Peñuela (2000) trabajó en un área de aguas abiertas y profundas ubicada a unos $20 \mathrm{~km}$ de la estación externa (Est. A). Por esto se puede señalar que las condiciones hidrográficas y biológicas varían de manera similar en zonas muy cercanas a la costa y en mar abierto.

En ambos análisis de componentes principales (ACP) los resultados muestran una tendencia similar. En ese sentido se observa la relación inversa y significativa entre la clorofila, temperatura, oxígeno disuelto y transparencia, todas del primer componente. Esto sugiere el efecto de surgencia costera de la región y que es dominante en relación a las tendencias anuales (Mandelli \& Ferraz-Reyes 1982, Ferraz-Reyes 1987). La ausencia de correlación entre la biomasa del fitoplancton y el zooplancton podría ser evidencia de la diferencia en escala temporal de los factores físicos y químicos (Mallin \& Paerl 1994, Franco-Gordo et al. 2004) y la tasa de pastoreo por parte del zooplancton herbívoro (Poullet et al. 2005). El zooplancton forma parte del segundo componente en el que la correlación es positiva y significativa entre el volumen desplazado y el peso seco.

Al comparar los resultados obtenidos para los factores bióticos y abióticos de 1997 y 1998 es evidente que existen fuertes variaciones anuales. La gran diferencia estacional de la biomasa observada en estos dos años apoya la necesidad del establecimiento de series de tiempo de larga duración. Ferraz-Reyes (1987) y Rueda (2000) también han señalado la existencia de grandes diferencias anuales en los parámetros bióticos $\mathrm{y}$ abióticos en el nororiente de Venezuela. Sin embargo, cambios drásticos en la biomasa han sido citados por Chávez et al. (2002), Hernández de la Torre et al. (2004), Franco-Gordo et al. (2004) en el Pacífico, frente a Baja California por el fenómeno ENOS (El Niño Oscilación del Sur) para
1997-1998. A pesar que se desconoce como afectan fenómenos como ENOS las condiciones oceanográficas del Mar Caribe suroriental; precisamente para ese año (1998) se observó en el presente trabajo un cambio drástico en el IS, un calentamiento de las aguas superficiales y una reducción notable de la biomasa fito- y macrozooplanctónica, evidenciándose posibles efectos en el área del Caribe.

Los parámetros de producción secundarios mostraron valores más bajos en las capas superiores que en las profundas en ambas estaciones y años. Una mayor concentración subsuperficial de los organismos planctónicos es normalmente encontrada en los cuerpos de agua $y$ es explicado por los procesos de concentración del plancton como respuesta migratoria y de evasión al mayor efecto deletéreo de la luz solar y el efecto turbulento de la superficie (Rueda 2000). Zoppi (1961) encontró una distribución similar en el Golfo de Cariaco, mientras que Urosa (1983) lo hizo en la cuenca de Cariaco.

La diferencia en la biomasa en áreas internas y externas de algunos cuerpos de agua, como la encontrada en la bahía de Mochima puede ser explicada por la batimetría. Zoppi (1961) indicó que en regiones costeras y en aguas de poca profundidad, la biomasa del zooplancton es mayor, debido a que los nutrientes terrígenos elevan la producción primaria en áreas cercanas a la costa. Además, la poca profundidad permite una distribución uniforme de los nutrientes en la columna por efecto de la turbulencia. De igual manera Cervantes-Duarte et al. (1993) y Gasca \& Castellanos (1993) encontraron mayor concentración de zooplancton en estaciones cercanas a la costa con respecto a las estaciones alejadas en San Hipólito (Baja California, México) y la bahía de Chetumal (Golfo de México), respectivamente. También, Rodríguez \& Rubin (1991) determinaron una mayor densidad ictioplanctónica en las estaciones más costeras en el sur de Galicia.

En general, la producción secundaria macro zooplanctónica de la bahía de Mochima durante el período de estudio fue muy baja en comparación con los valores obtenidos en otras regiones de Venezuela y del mundo. Los valores más elevados registrados en este estudio están en el límite inferior de los reportados por Rao \& Urosa (1974) para el Golfo de Cariaco (volumen desplazado, 0,02-0,28 $\mathrm{mL} \mathrm{m}^{-3}$ ) y por Urosa (1983) y Peñuela (2000) en la cuenca de Cariaco (peso seco, 2,8 g $100 \mathrm{~m}^{-3}$ y volumen desplazado, 0,63 $\mathrm{mL} \mathrm{m}^{-3}$, 
respectivamente). Por otra parte, González \& Alfonso (1982), Jiménez-Pérez (1989) y Jiménez \& Lara (1990) reportaron valores entre 8 y $250 \mathrm{mg} \mathrm{m}^{-3}$ de peso seco; 0,08 y $0,78 \mathrm{~mL} \mathrm{~m}^{-3}$ y 0,09 a $0,78 \mathrm{~mL} \mathrm{~m}^{-3}$ de volumen desplazado en la ensenada de la Broa (costa suroccidental de Cuba), en la bahía de Todos Santos y en el estero de Punta Banda (México), respectivamente.

La diferencia en la frecuencia de los muestreos y en las horas de captura podrían explicar por qué los valores de biomasa macrozooplanctónica obtenidos en este trabajo son bajos en comparación con los citados por Rao \& Urosa (1974), Urosa (1983) y Peñuela (2000). El efecto de la frecuencia muestreal es un factor muy importante en la determinación de la productividad de los diferentes eslabones de la cadena trófica. Recientemente Müller-Karger et al. (2000), utilizando las mediciones de productividad primaria de la serie de tiempo CARIACO, encontraron que había grandes diferencias en la productividad primaria de la cuenca de Cariaco. Las mediciones históricas realizadas de manera esporádica informaban sobre valores de 200 a $400 \mathrm{gC} \mathrm{m}^{2}$ año $^{-1}$ mientras que los obtenidos en la estación CARIACO a partir de mediciones mensuales continuas desde noviembre de 1995 estaban en el orden de $>500 \mathrm{gC} \mathrm{m}^{2}$ año ${ }^{-1}$.

\section{Agradecimientos}

Los autores quieren hacer un reconocimiento a la colaboración brindada por: Luís E. González, Alexander Barrios, Luís Ortega y Carlos Moreno. Igualmente agradecemos el aporte de los evaluadores anónimos. Este trabajo fue financiado por el Consejo de Investigación de la Universidad de Oriente (Proyecto CI-5-1803-0850/99). La primera autora agradece todo el apoyo del Fondo Nacional de Ciencia, Tecnología e Innovación (FONACIT) a través del Programa de Investigador Novel (PIN).

\section{Literatura citada}

Beers JR. 1981. Determinación de la biomasa del zooplancton. En: Boltovskoy D (ed). Atlas del Zooplancton del Atlántico Sudoccidental y Métodos de Trabajo con el Zooplancton Marino, pp. 133-140. Publicación especial del INIDEP, Mar de Plata, Argentina.

Bowden KF. 1983. Physical oceanography of coastal waters, 302 pp. Ellis Horwood Ltd., Chichester.
Cervantes-Duarte R, SA García \& SH Trujillo. 1993. Condiciones de surgencia asociadas a la distribución de zooplancton en San Hipólito, B.C.S. Ciencias Marinas 19(1): 117-135.

Clark D, KV Aazem \& GC Hays. 2001. Zooplankton abundance and community structure over a $4000 \mathrm{Km}$ transect in the north-east Atlantic. Journal of Plankton Research 23 (4): 365-372.

Chavez FP, JT Pennington, CG Castro, JP Ryan, RP Mishisaki, B Schlining, P Walz, KR Buck, A McFadyen \& CA Collins. 2002. Biological and chemical consequences of the 1997-1998 El Niño in central California waters. Progress in Oceanography 54(1-4): 205-232.

Expósito N \& E Zoppi. 1999. Efectos de las descargas de una laguna de estabilización sobre el ecosistema marino. Libro de Resúmenes Ampliados del VIII Congreso Latinoamericano sobre Ciencias del Mar (COLACMAR), Perú, Tomo II: 845-847.

Fernández de Puelles M \& JA García-Braun. 1989. Dinámica de las comunidades planctónicas en una zona del Atlántico subtropical (Isla de Tenerife). Boletín del Instituto Español de Oceanografía 5(2): 87-100.

Fernández de Puelles M. 1990. Evolución temporal de la biomasa zooplanctónica en el Mar Balear. Boletín del Instituto Español de Oceanografía 6(1): 95-106.

Ferraz-Reyes E. 1987. Productividad primaria del Golfo de Cariaco, Venezuela. Boletín del Instituto Oceanográfico de Venezuela, Universidad de Oriente 26 (1/2): 97-110.

Franco-Gordo C, E Godínez-Domínguez, AE Filonov, IE Tereshchenko \& J Freire. 2004. Plankton biomass and larval fish abundance prior to and during the El Niño period of 1997-1998 along the central Pacific coast of México. Progress in Oceanography 63: 99-123.

Gasca R \& I Castellanos. 1993. Zooplancton de la Bahía de Chetumal, Mar Caribe, México. Revista de Biología Tropical 41(3): 619-625.

Gasca R \& E Suárez. 1996. Introducción al estudio del zooplancton marino, $711 \mathrm{pp}$. El colegio de la Frontera Sur (ECOSUR), Chetumal, México.

García A. 1978. Observación de algunos parámetros ambientales de la Bahía de Mochima durante el período de enero - junio de 1977. Tesis Magíster Scientarium en Biología Marina. Universidad de Oriente, Venezuela, 59 pp.

González E \& I Alfonso. 1982. Caracterización cuantitativa y cualitativa del zooplancton en la Ensenada de la Broa. Revista Cubana de Investigaciones Pesqueras 7(3): 47-63. 
González F \& E Zoppi. 1997. Distribución espacial del fitoplancton y pigmentos fotosintéticos en la Bahía de Mochima, Libro de Resúmenes Ampliados del VII Congreso Latinoamericano sobre Ciencias del Mar (COLACMAR), Brasil, Tomo I: 396-398.

Griffiths R \& J Simpson. 1972. Afloramiento y otras características oceanográficas de aguas costeras del nordeste de Venezuela. MAC. Series Recursos Explotables Pesqueros 1: 2-53.

Guzmán H \& V Obando. 1988. Diversidad y abundancia diaria y estacional del zooplancton marino de la Isla del Caño, Costa Rica. Revista de Biología Tropical 36(1): 139-150.

Hernández de la Torre B, G Gaxiola-Castro \& S NájeraMartínez. 2004. Efectos del ENSO en la producción primaria frente a Baja California. Ciencias Marinas 30(3): 427-441.

Jiménez-Pérez LC. 1989. Variación temporal del zooplancton de la Bahía de Todos Santos, Baja California, México. Ciencias Marinas 15(3): 81-96.

Jiménez PL \& JR Lara. 1990. Distribución de biomasa y estructura de la comunidad del zooplancton en el Estero de Punta Banda. Ciencias Marinas 16(1): 35-48.

Johnson R \& D Wichern. 1992. Applied multivariate statistical analysis. 642 pp. Prentice-Hall, Nueva York.

Kato K. 1972. Some aspects on biochemical characteristics of sea and sediments in Mochima Bay, Venezuela. Boletín del Instituto Oceanográfico de Venezuela, Universidad de Oriente 1: 343-358.

Lodeiros C \& J Himmelman. 1994. Relations among environmental conditions and growth in the tropical scallop Euvola (Pecten) ziczac (L.) in suspended culture in the Golfo de Cariaco, Venezuela. Aquaculture 119: 345-358.

McEwen GF, MW Johnson \& TR Folsom. 1954. A statistical analysis of the performance of the Folsom Plankton Splitter based upon test observations. Archiv für Meteorologie. Geophysik und Bioklimatologie Ser A: Meteorologie und Geophysik 1(17):167-175.

Mallin M \& H Paerl. 1994. Planktonic trophic transfer in an estuary: Seasonal diel and community structure effects. Ecology 75(8): 2168-2184.

Mandelli E \& E Ferraz-Reyes. 1982. Primary production and phytoplankton dynamics in a tropical inlet, Gulf of Cariaco, Venezuela. International Review of Hydrobiology 67(1): 85-95

Moigis AG. 1986. Variación anual de la productividad primaria del fitoplancton en el Golfo y en la Fosa de Cariaco, Venezuela. Boletín del Instituto Oceanográfico de Venezuela, Universidad de Oriente 25: 115-126.
Muller-Karger F, $\mathbf{R}$ Varela, $\mathbf{R}$ Thunell, $\mathbf{M}$ Scranton, $\mathbf{R}$ Bohrer, G Taylor, J Capelo, Y Astor, E Tappa, T-Y Ho, M Iabichella, J Walsh \& JR Díaz. 2000. Sediment record linked to surface processes in the Cariaco Basin. EOS, Transactions of the American Geophysical Union 81(45): 534-535.

Okuda T, J Benítez, A García \& E Fernández. 1968. Condiciones hidrográficas y químicas en la Bahía de Mochima y la Laguna Grande del Obispo desde 1964 a 1966. Boletín del Instituto Oceanográfico de Venezuela, Universidad de Oriente 7(2): 7-38.

Okuda T \& J Benítez. 1974. Condiciones hidrográficas de las capas superiores en la Fosa de Cariaco y áreas adyacentes durante la época lluviosa. Boletín del Instituto Oceanográfico de Venezuela, Universidad de Oriente 13 (1/2): 147-162.

Peñuela M. 2000. Análisis cuantitativo y cualitativo del zooplancton superficial de la Fosa de Cariaco, Venezuela. Tesis de Grado, Departamento de Biología, Escuela de Ciencias, Universidad de Oriente, Venezuela, 100 pp.

Postel L, H Fock \& W Hagen. 2000. Biomass and abundance. En: Harris RP, PH Wiebe, J Lenz, HR Skjoldal \& M Huntley (eds). Ices Zooplankton Methodology Manual, pp. 83-174. San Diego, California.

Poulet S, L Mohamed \& Y Chaudron. 2005. Characteristic features of zooplankton in the Bay of Biscay. Scientia Marina 60 (Suppl. 2): 79-95

Rao T \& LJ Urosa. 1974. Ecología del zooplancton en el Golfo de Cariaco. Parte I. Variabilidad de la biomasa durante el período de agosto a noviembre de 1973. Boletín del Instituto Oceanográfico de Venezuela, Universidad de Oriente 13 (1/2): 67-78.

Richards F. 1960. Some chemical and hydrographic observations along the north coast of South America 1. Cabo Tres Puntas to Curacao, including the Cariaco Trench and the Gulf of Cariaco. Deep Sea Research 7: 163-182.

Rodríguez J \& J Rubín. 1991. El ictioplancton y la biomasa del zooplancton en aguas del sur de Galicia, en abril 1987. Boletín del Instituto Español de Oceanografía 7(2): 115-127.

Rueda D. 2000. Variabilidad temporal de la distribución vertical de la biomasa fitoplanctónica en la depresión oriental de la cuenca de Cariaco y sus relaciones con los aspectos hidrográficos del estrato superficial (19961998). Tesis Magíster Scientarium en Biología Marina. Universidad de Oriente, Venezuela, 120 pp.

Sokal JD \& FJ Rohlf. 1981. Biometry, 859 pp. WH Freeman and Company, Nueva York. 
Strickland J \& T Parsons. 1972. Manual of sea water analysis. Bulletin Fisheries Research Board of Canada. $125,310 \mathrm{pp}$.

Urosa L. 1977. Bibliografía y observaciones sobre el zooplancton del mar Caribe. Lagena 39-40: 11-26.

Urosa LJ. 1983. Distribución del zooplancton en la cuenca Tuy-Cariaco, área de posible actividad petrolera en Venezuela. Boletín del Instituto Oceanográfico de Venezuela, Universidad de Oriente 22 (1/2): 125-143.

Zoppi E. 1961. Distribución vertical del zooplancton en el Golfo y extremo este de la Fosa de Cariaco. Boletín del Instituto Oceanográfico de Venezuela, Universidad de Oriente 1(1): 219-248.
Zoppi de Roa E, P Alonso \& M Delgado. 1989. Distribución de los Misidáceos (Crustacea) en las costas de Venezuela. Boletín del Instituto Oceanográfico de Venezuela, Universidad de Oriente 28 (1/2): 29-33.

Zoppi de Roa E. \& P Alonso. 1997. Distribution of Mysidium integrum (Tattersall) (Crustacea-Mysidacea) in Venezuelan coral habitats. Studies on the Natural History of the Caribbean Region 73: 1-15.

Zoppi de Roa E. 1999. El zooplancton de la zona interna de la Bahía de Mochima, Venezuela. Libro de Resúmenes Ampliados del VIII Congreso Latinoamericano sobre Ciencias del Mar (COLACMAR). Tomo I, 354 pp. Perú.

Recibido el 16 de marzo de 2007 y aceptado el 9 de julio de 2007 